\title{
Comparison of the Clinical Outcomes of Patients with Squamous Cell Carcinoma of the Tonsil Receiving Postoperative Ipsilateral Versus Bilateral Neck Radiotherapy: A Propensity Score Matching Analysis (KROG 11-07)
}

\section{Youngkyong Kim, MD \\ Kwan Ho Cho, MD' \\ Sung Ho Moon, MD ${ }^{1}$ \\ Chang Geol Lee, MD2 \\ Ki Chang Keum, MD2 \\ Sang-wook Lee, $\mathrm{MD}^{3}$ \\ Yong Chan Ahn, $\mathrm{MD}^{4}$ \\ Dongryul Oh, MD ${ }^{4}$ \\ Yeon-Sil Kim, MD ${ }^{5}$ \\ Yong Kyun Won, MD \\ Hong-Gyun Wu, MD \\ J. Hun Hah, MD \\ Young-Taek Oh, MD}

${ }^{1}$ Proton Therapy Center, National Cancer Center, Goyang, ${ }^{2}$ Department of Radiation Oncology, Yonsei University College of Medicine, Seoul, ${ }^{3}$ Department of Radiation Oncology, Asan Medical Center, University of Ulsan College of Medicine, Seoul,

${ }^{4}$ Department of Radiation Oncology, Samsung Medical Center, Sungkyunkwan University School of Medicine, Seoul, ${ }^{5}$ Department of Radiation Oncology, Seoul St. Mary's Hospital, College of Medicine, The Catholic University of Korea, Seoul, Departments of ${ }^{6}$ Radiation Oncology and ${ }^{7}$ Otolaryngology-Head and Neck Surgery, Seoul National University College of Medicine, Seoul, ${ }^{8}$ Department of Radiation Oncology, Ajou University School of Medicine, Suwon, Korea

Correspondence: Kwan Ho Cho, MD Proton Therapy Center, National Cancer Center, 323 Ilsan-ro, Ilsandong-gu, Goyang 10408, Korea Tel: 82-31-9201720

Fax: 82-31-9200149

E-mail: kwancho@ncc.re.kr

Received September 3, 2016

Accepted January 23, 2017

Published Online February 9, 2017

*Presented as an e-poster discussion session at the 2015 Annual Meeting of the American Society for Radiation Oncology, October 18-21, 2015, San Antonio, Texas, USA.

\section{Purpose}

The impact of postoperative ipsilateral neck radiotherapy (INRT) versus bilateral neck radiotherapy (BNRT) on the clinical outcomes of patients with tonsillar squamous cell carcinoma was analyzed retrospectively.

\section{Materials and Methods}

Between October 2001 and June 2012, 241 patients with T1-2 and NO-N2b tonsillar carcinoma from 16 institutes underwent postoperative INRT ( $n=84)$ or BNRT $(n=157)$ following a tonsillectomy. Seventy patients were identified from each group by propensity score matching and compared in terms of the overall survival (OS), disease-free survival (DFS), locoregional relapse-free survival (LRRFS), and distant metastasis-free survival (DMFS) rates calculated using the Kaplan-Meier method with a log-rank test.

\section{Results}

The median follow-up was 55 months (range, 3 to 133 months). The survival outcomes in the INRT and BNRT groups were similar: 5-year OS (92.8\% vs. 94.0\%, p=0.985), DFS (80.5\% vs. $94.2 \%$. $p=0.085)$, LRRFS (88.1\% vs. $97.1 \%, p=0.083)$, and DMFS $(92.7 \%$ vs. $97.0 \%$, $\mathrm{p}=0.370$ ). Subgroup analysis revealed no contralateral neck recurrence in 61 patients with T1-2NO-2a regardless of the treatment groups. For 79 patients with N2b, contralateral neck recurrence was more common in the INRT group than in the BNRT group (7.9\% vs. 0.0\%), but the difference was not significant $(p=0.107)$. The overall grade $\geq 2$ toxicities were lower in the INRT group: acute ( $45.7 \%$ vs. $74.3 \%, p=0.001)$ and late $(4.3 \%$ vs. $31.4 \%, p<0.001)$, respectively.

\section{Conclusion}

INRT is an attractive strategy for patients with T1-2NO-2a tonsillar carcinoma compared to BNRT. For patients with N2b, there was a small risk of contralateral neck recurrence when treated with INRT, but its impact on the OS was limited with successful salvage treatment.

\section{Key words}

Ipsilateral neck, Adjuvant radiotherapy, Tonsillar neoplasms, Radiation volume, Toxicity 


\section{Introduction}

In carcinoma of the oropharyngeal region, the area treated with radiation conventionally includes both cervical lymphatic chains, regardless of nodal involvement, because metastasis to the regional lymph nodes is reported frequently, even in clinically node-negative tumors [1,2]. This extensive radiation-targeted volume contributes to favorable locoregional control in tonsil cancer but it may also lead to treatment-related morbidity, such as xerostomia, dysphagia, and mucositis. Permanent xerostomia is one of the most common and distressing complications after radiation treatment for head and neck cancer. Intensity-modulated radiotherapy (IMRT) is a good strategy for preventing xerostomia because the salivary glands can be spared [3]. Although IMRT reduces long-term severe xerostomia compared to threedimensional conformal radiotherapy (3D-CRT), other sequelae, including the skin and mucosal toxicity, as well as low grade xerostomia can persist after IMRT [4,5]. In addition, patients treated unilaterally achieve faster recovery and higher salivary function relative to the pretreatment levels than those treated bilaterally by IMRT $[5,6]$.

Several institutes have reported their experience of using a limited radiation field for patients with well-lateralized cancers of the tonsillar fossa to exclude treating the uninvolved contralateral neck; the contralateral regional recurrence rates were $<6 \%$ [7-11]. These findings suggest that not all patients are likely to benefit from prophylactic radiation encompassing the contralateral neck. Recently, there is an increasing trend of younger patients with oropharyngeal cancer usually associated with the human papillomavirus (HPV) [12]. The treatment outcomes for HPV-related oropharyngeal cancer are superior to those of non-HPV-related disease $[13,14]$. These evolving trends could also support a de-intensification strategy through a reduction of the radiation volume. Although the American College of Radiology provided recommendations for ipsilateral neck radiotherapy (INRT) based on published retrospective data, there are no reports comparing the survival outcomes and failure patterns between INRT and bilateral neck radiotherapy (BNRT).

This study analyzed retrospectively the clinical outcomes based on the radiotherapy (RT) area, for INRT versus BNRT, in patients who underwent a tonsillectomy for the treatment of tonsillar cancer who were matched using a propensityscoring method. The investigation was a multi-institutional, retrospective study of the Korean Radiation Oncology Group, 11-07.

\section{Materials and Methods}

\section{Patients}

The medical records of patients with tonsillar carcinoma, who received a tonsillectomy followed by adjuvant RT between October 2001 and June 2012 at 16 institutes in Korea, were reviewed retrospectively. The eligibility criteria for the present study included (1) histologically confirmed squamous cell carcinoma in the tonsillar region, (2) non-distant metastatic disease status at diagnosis, (3) postoperative treatment with 3D-CRT or IMRT, (4) age $\geq 20$ years, (5) Eastern Cooperative Oncology Group (ECOG) performance status $0-2$, and (6) no previous radiation of the head and neck region. Of 390 patients, 149 were excluded from the analyses for the following reasons: advanced disease, which is classified as T3-4 or N3 ( $\mathrm{n}=88)$; bilateral neck lymph nodes involvement $(\mathrm{n}=37)$; incomplete treatment $(\mathrm{n}=2)$; and insufficient data for analysis $(n=22)$. Ultimately, 241 patients were included in the analysis.

Of these 241 patients, seventy patients were identified from each group (INRT and BNRT) by propensity score matching (PSM) based on their smoking history, chemotherapy, extranodal extension, $\mathrm{T} / \mathrm{N}$ classification, and performance status. After PSM, the patients in each group were comparable in terms of the potential prognostic indicators. Table 1 lists the patient characteristics before and after PSM. All patients underwent pretreatment workup comprising a physical examination, routine laboratory testing (complete blood count, liver function test), chest radiography, and neck magnetic resonance imaging or computed tomography. ${ }^{18} \mathrm{~F}$ deoxyfluoroglucose positron emission tomography-computed tomography and neck ultrasonography were also applied when necessary.

\section{Treatments}

The extent and radiation dose were determined by the policies of each institution. The clinical target volume (CTV) was defined as follows: CTV1, primary tumor bed and the neck level of involved nodes; CTV2, adjacent neck level of involved nodes; and CTV3, elective region of the uninvolved neck. The planning target volume (PTV) was defined as the CTV plus $0.3-1.0 \mathrm{~cm}$ in all directions. For INRT, the median total doses applied to PTV1, PTV2, and PTV3 were 60.0 Gy (range, 54.0 to $70.0 \mathrm{~Gy}$ ), $59.4 \mathrm{~Gy}$ (range, 36.0 to $70.0 \mathrm{~Gy}$ ), and 45.0 Gy (range, 36.0 to $54.0 \mathrm{~Gy}$ ), respectively, with a daily dose of 1.71 to $2.25 \mathrm{~Gy}$. For BNRT, the median total doses applied to PTV1, PTV2, and PTV3 were 63.0 Gy (range, 50.0 to $72.0 \mathrm{~Gy}$ ), 54.0 Gy (range, 27.0 to $66.0 \mathrm{~Gy}$ ), and 50.0 Gy (range, 30.6 to 60.0 Gy), respectively, with a daily dose of 1.6- 
Table 1. Patients' characteristics before and after propensity-score matching

\begin{tabular}{|c|c|c|c|c|c|c|}
\hline \multirow{2}{*}{ Characteristic } & \multicolumn{3}{|c|}{ Total population } & \multicolumn{3}{|c|}{ Propensity score-matched population } \\
\hline & INRT (n=84) & BNRT $(n=157)$ & p-value ${ }^{a}$ & INRT $(n=70)$ & BNRT $(n=70)$ & p-value $\left.{ }^{a}\right)$ \\
\hline \multicolumn{7}{|l|}{ Age (yr) } \\
\hline Median (range) & $54(31-76)$ & $54(32-81)$ & & $55(31-76)$ & $55(32-81)$ & \\
\hline$<60$ & $58(69.0)$ & $113(72.0)$ & 0.633 & $48(68.6)$ & $50(71.4)$ & 0.712 \\
\hline$\geq 60$ & $26(31.0)$ & $44(28.0)$ & & $22(31.4)$ & $20(28.6)$ & \\
\hline \multicolumn{7}{|l|}{ Sex } \\
\hline Male & $73(86.9)$ & $141(89.8)$ & 0.496 & $61(87.1)$ & $62(88.6)$ & 0.796 \\
\hline Female & $11(13.1)$ & $16(10.2)$ & & $9(12.9)$ & $8(11.4)$ & \\
\hline \multicolumn{7}{|l|}{ Histologic grade } \\
\hline WD & $14(16.7)$ & $17(10.8)$ & 0.087 & $9(12.9)$ & $5(7.1)$ & 0.348 \\
\hline MD & $45(53.6)$ & $96(61.1)$ & & $39(55.7)$ & $45(64.3)$ & \\
\hline PD & $17(20.2)$ & $39(24.8)$ & & $15(21.4)$ & $17(24.3)$ & \\
\hline NA & $8(9.5)$ & $5(3.2)$ & & $7(10.0)$ & $3(4.3)$ & \\
\hline \multicolumn{7}{|l|}{ HPV } \\
\hline$(+)$ & $5(6.0)$ & $11(7.0)$ & 0.526 & $4(5.7)$ & $5(7.1)$ & 0.807 \\
\hline$(-)$ & $3(3.6)$ & $2(1.3)$ & & $3(4.3)$ & $1(1.4)$ & \\
\hline NA & $76(90.5)$ & $144(91.7)$ & & $63(90.0)$ & $64(91.4)$ & \\
\hline \multicolumn{7}{|l|}{ Smoking } \\
\hline Never smoking & $44(52.4)$ & $56(35.7)$ & $0.010^{*}$ & $36(51.4)$ & $33(47.1)$ & 0.536 \\
\hline Yes, but quit & $19(22.6)$ & $27(17.2)$ & & $13(18.6)$ & $18(25.7)$ & \\
\hline Current smoker & $16(19.0)$ & $59(37.6)$ & & $16(22.9)$ & $17(24.3)$ & \\
\hline NA & $5(6.0)$ & $15(9.6)$ & & $5(7.1)$ & $2(2.9)$ & \\
\hline \multicolumn{7}{|c|}{ Extracapsular extension } \\
\hline$(+)$ & $22(26.2)$ & $48(30.6)$ & 0.766 & $22(48.6)$ & $18(25.7)$ & 0.594 \\
\hline$(-)$ & $46(54.8)$ & $82(52.2)$ & & $34(31.4)$ & $40(57.1)$ & \\
\hline NA & $16(19.0)$ & $27(17.2)$ & & $14(20.0)$ & $12(17.1)$ & \\
\hline \multicolumn{7}{|l|}{ T classification } \\
\hline 1 & $29(34.5)$ & $55(35.0)$ & 0.937 & $23(32.9)$ & $25(35.7)$ & 0.722 \\
\hline 2 & $55(65.5)$ & $102(65.0)$ & & $47(67.1)$ & $45(64.3)$ & \\
\hline \multicolumn{7}{|l|}{$\mathbf{N}$ classification } \\
\hline 0 & $20(23.8)$ & $14(8.9)$ & $<0.001^{*}$ & $10(14.3)$ & 10 (14.3) & 0.953 \\
\hline 1 & $20(23.8)$ & $20(12.7)$ & & $16(22.9)$ & $14(20.0)$ & \\
\hline $2 a$ & $6(7.1)$ & $11(7.0)$ & & $6(8.6)$ & $5(7.1)$ & \\
\hline $2 b$ & $38(45.2)$ & $112(71.3)$ & & $38(54.3)$ & $41(58.6)$ & \\
\hline \multicolumn{7}{|l|}{ Staging } \\
\hline I & $5(6.0)$ & $1(0.6)$ & $<0.001^{*}$ & $3(4.3)$ & $1(1.4)$ & 0.751 \\
\hline II & 15 (17.9) & $13(8.3)$ & & $7(10.0)$ & 9 (12.9) & \\
\hline III & $20(23.8)$ & $20(12.7)$ & & $16(22.9)$ & $14(20.0)$ & \\
\hline IVA & $44(52.4)$ & $123(78.3)$ & & $44(62.9)$ & $46(65.7)$ & \\
\hline \multicolumn{7}{|l|}{ Chemotherapy } \\
\hline Yes & $18(21.4)$ & $50(31.8)$ & $0.029^{*}$ & $17(24.3)$ & $18(25.7)$ & 0.845 \\
\hline No & $66(78.6)$ & 107 (68.2) & & $53(75.7)$ & $52(74.3)$ & \\
\hline \multicolumn{7}{|l|}{ ECOG PS } \\
\hline 0 & $18(21.4)$ & $60(38.2)$ & $0.002^{*}$ & $18(25.7)$ & 15 (21.4) & 0.803 \\
\hline 1 & $64(76.2)$ & $83(52.9)$ & & $50(71.4)$ & $52(74.3)$ & \\
\hline 2 & 0 & $6(3.8)$ & & 0 & 0 & \\
\hline NA & $2(2.4)$ & $8(5.1)$ & & $2(2.9)$ & $3(4.3)$ & \\
\hline
\end{tabular}

Values are presented as number (\%). INRT, ipsilateral neck radiotherapy; BNRT, bilateral neck radiotherapy; WD, well differentiated; MD, moderately differentiated; PD, poorly differentiated; NA, not available; HPV, human papillomavirus; ECOG, Eastern Cooperative Oncology Group; PS, performance status. *Statistical significance $(\mathrm{p}<0.05)$. ${ }^{a}$ Chi-square or Fisher exact test. 
A
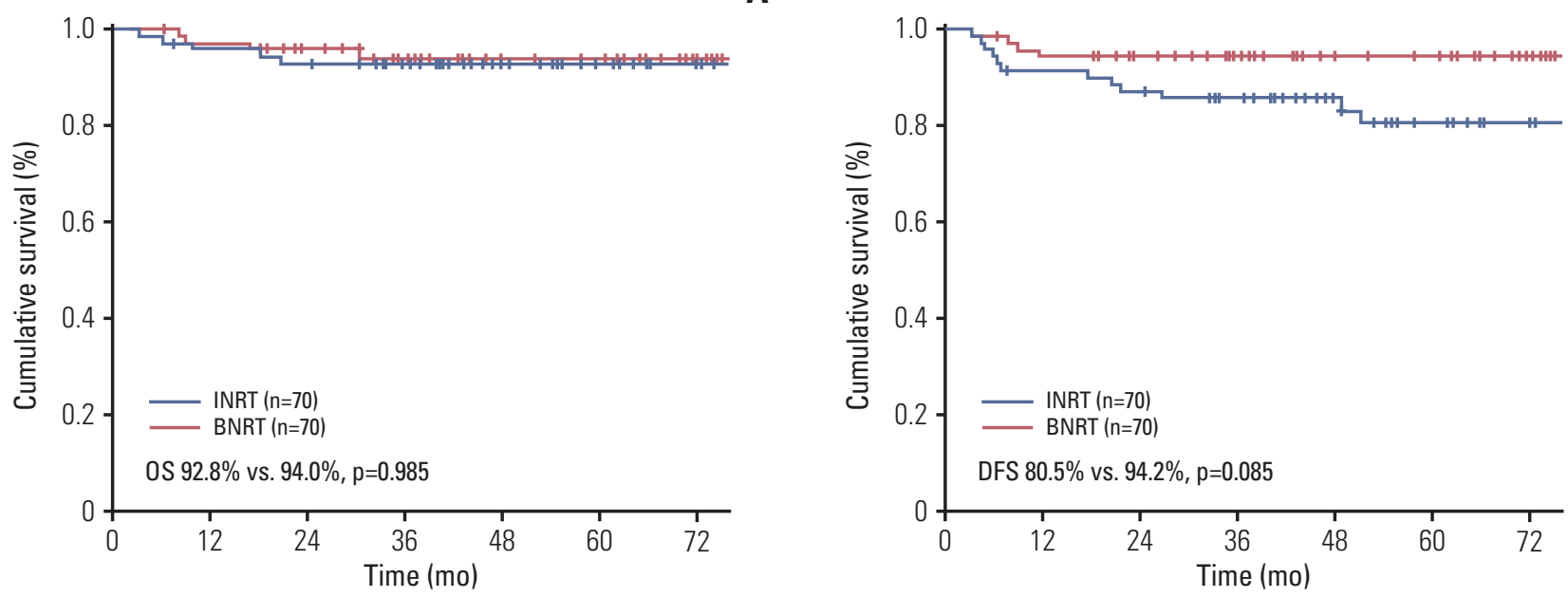

C
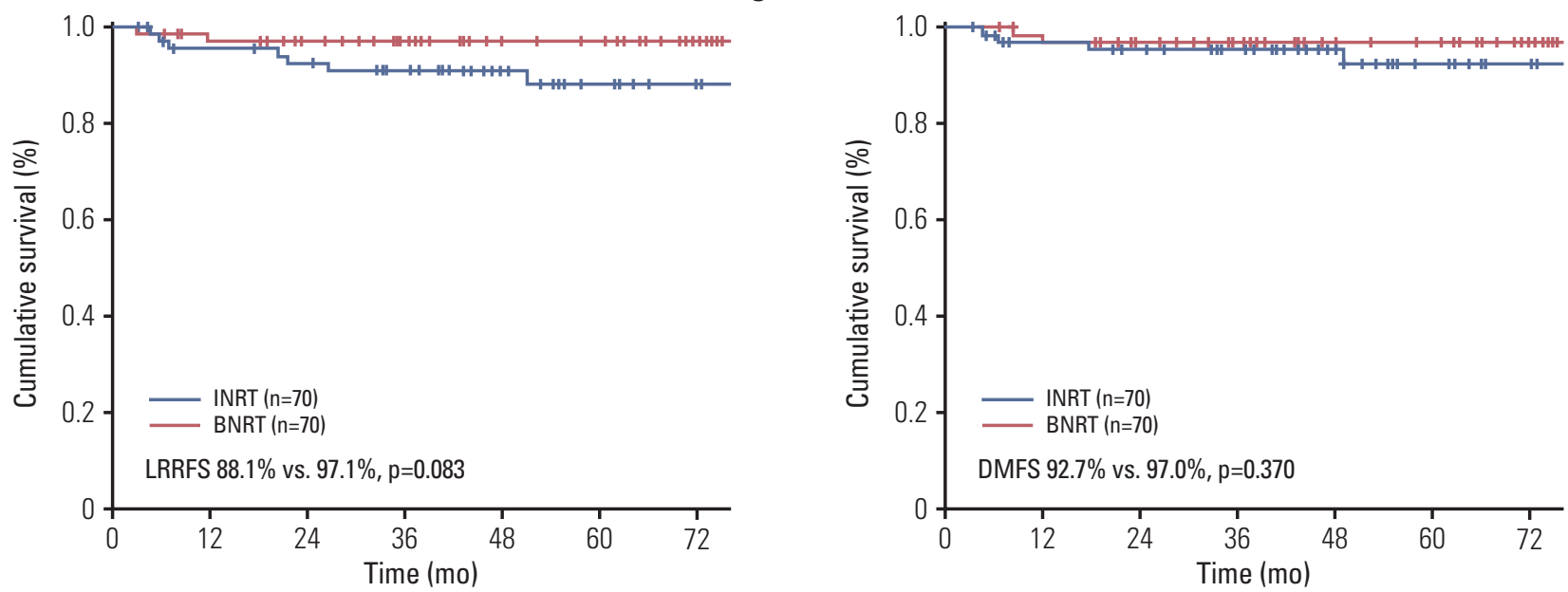

Fig. 1. Comparison of the 5-year overall survival (OS) (A), disease-free survival (DFS) (B), locoregional relapse-free survival (LRRFS) (C), and distant metastasis-free survival (DMFS) (D) rates between the ipsilateral neck radiotherapy (INRT) and bilateral neck radiotherapy (BNRT) groups defined by propensity-score matching.

2.4 Gy. More patients in the BNRT group received IMRT than in the INRT group $(31 / 70,44.2 \%$ vs. $5 / 70,7.1 \%$, respectively). A neck dissection was performed in $59(84.3 \%)$ and $55(78.6 \%)$ patients in the INRT and BNRT groups, respectively. Chemotherapy was administered sequentially or concurrently with regimens, including cisplatin, carboplatin, or cetuximab.

\section{Statistical analysis}

The patients were allocated to either the INRT or BNRT group based on whether or not the radiation volume encom- passed the uninvolved contralateral neck. The baseline characteristics of the patients were compared using chi-sqaure or Fisher exact tests. PSM was conducted by modeling the probability of the patients in both the INRT and BNRT groups. The probability of each individual patient was estimated using a logistic regression model based on their smoking history, administration of chemotherapy, extranodal extension, $\mathrm{T} / \mathrm{N}$ classification, and ECOG performance status. The events of locoregional recurrence-free survival (LRRFS), distant metastasis-free survival (DMFS), disease-free survival (DFS), and overall survival (OS) were defined as locoregional recurrence, distant metastasis, any recurrence or death, and 

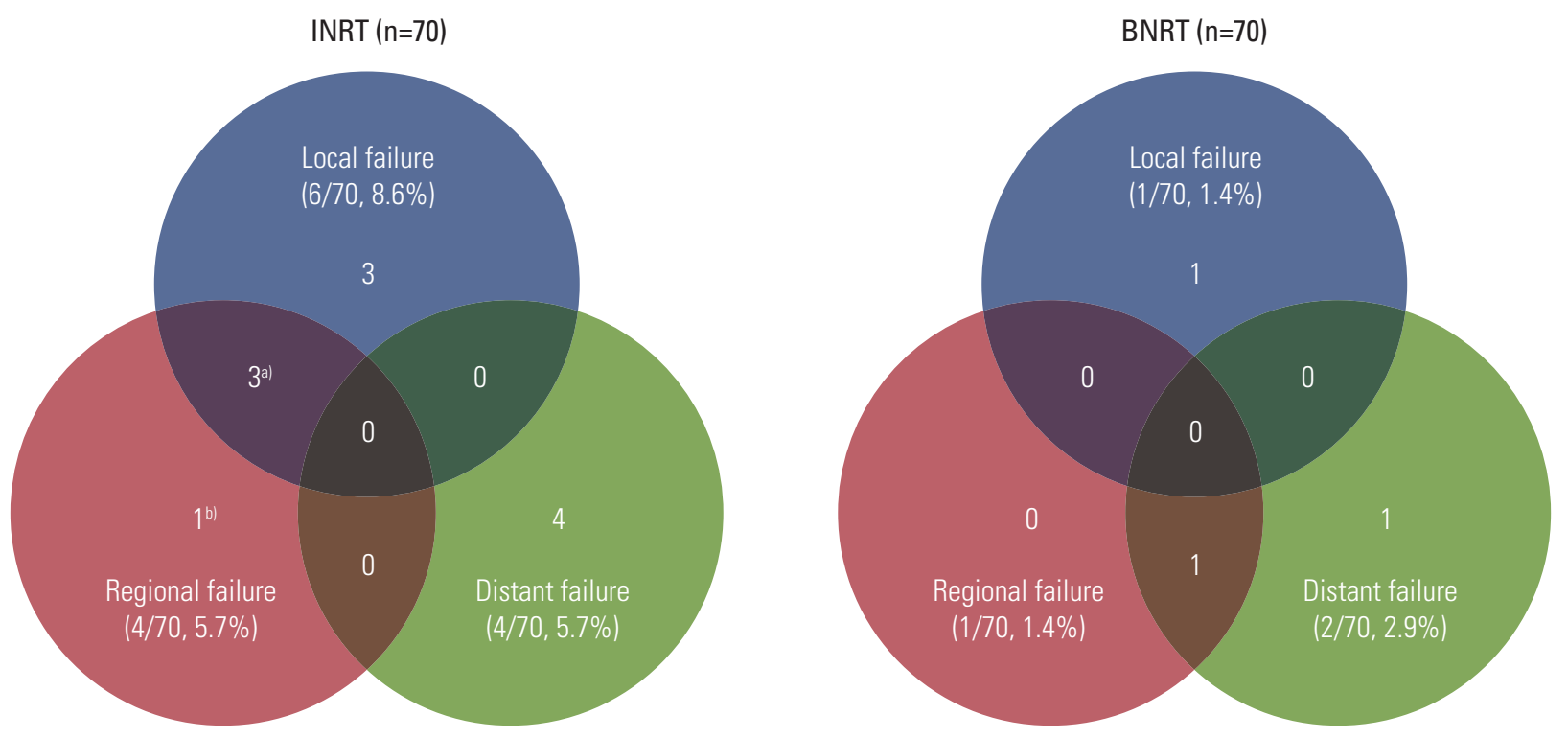

Median follow-up 55 mo (range, 3-133 mo)

Fig. 2. Patterns of failure in the ipsilateral neck radiotherapy (INRT) and bilateral neck radiotherapy (BNRT) groups defined

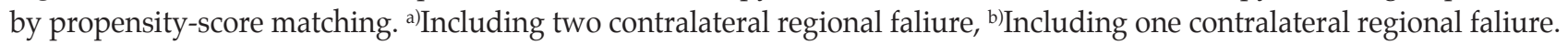

death, respectively. The survival rates were calculated from the initial date of treatment (tonsillectomy) to the date of each event or the last follow-up and were compared using the Kaplan-Meier method and log-rank test. The treatmentrelated toxicities were graded using the Radiation Therapy Oncology Group acute and late morbidity scoring system [15]. Acute toxicity was defined as occurring within 90 days of the initiation of treatment with toxicity considered late. The rates of toxicities in the two groups were compared using chi-square or Fisher exact tests. In all tests, p-values of $<0.05$ were considered significant.

\section{Results}

\section{Survival and patterns of failure}

The median follow-up duration of the PSM population was 55 months (range, 3 to 133 months). The 5-year LRRFS ( $88.1 \%$ vs. $97.1 \%, \mathrm{p}=0.083)$ and DFS $(80.5 \%$ vs. $94.2 \%$, $\mathrm{p}=0.085$ ) rates were higher in the BNRT group than in the INRT group with borderline significance. No significant differences in the DMFS (92.7\% vs. $97.0 \%, \mathrm{p}=0.370)$ and OS ( $92.8 \%$ vs. $94.0 \%, \mathrm{p}=0.985)$ were observed (Fig. 1 ). Fig. 2 pres- ents the patterns of failure. A total of 11 patients (15.7\%) in the INRT group experienced recurrence, whereas only three patients $(4.3 \%)$ experienced recurrence in the BNRT group. In the INRT group, three (4.3\%) and one (1.4\%) patient had isolated local and regional recurrence, respectively, three $(4.3 \%)$ had locoregional recurrence, and four $(5.7 \%)$ experienced distant recurrence. In the BNRT group, one patient each experienced local, distant, and regional with distant recurrences. Three patients (4.3\%) and no patients had contralateral regional recurrence in the INRT and BNRT group, respectively, but the difference between the groups was not statistically significant $(\mathrm{p}=0.245)$. The three patients in the INRT group with contralateral regional recurrence included one with an isolated regional recurrence and two with regional combined with local recurrence.

In subgroup analysis, there were no contralateral neck recurrences in patients with T1-2N0-2a regardless of the treatment groups, INRT or BNRT. For patients with N2b, contralateral neck recurrence was more common in the INRT group than in the BNRT group (3/38 patients [7.9\%] vs. $0 / 41$ patients $[0.0 \%]$, respectively), but the difference was not statistically significant $(\mathrm{p}=0.107)$. There was also a difference in the 5-year LRRFS ( $84.8 \%$ vs. $94.3 \%$, $\mathrm{p}=0.234$ ), but no statistical significance was identified. The 5-year OS ( $92.0 \%$ vs. $89.6 \%$, $\mathrm{p}=0.750$ ) was similar in the two groups after salvage treatment. 
Table 2. Acute and late toxicities of the patients treated with INRT and BNRT

\begin{tabular}{|c|c|c|c|c|}
\hline Toxicity & Grade $^{a)}$ & INRT $(n=70)$ & BNRT $(n=70)$ & p-value ${ }^{\text {b) }}$ \\
\hline \multicolumn{5}{|l|}{ Acute } \\
\hline \multirow[t]{4}{*}{ Xerostomia } & 0 & 32 (45.7) & $11(15.7)$ & $<0.001^{*}$ \\
\hline & 1 & 29 (41.4) & $32(45.7)$ & \\
\hline & 2 & $8(11.4)$ & $25(35.7)$ & \\
\hline & 3 & $1(1.4)$ & $2(2.9)$ & \\
\hline \multirow[t]{4}{*}{ Oral mucositis } & 0 & $10(14.3)$ & $7(10.0)$ & $0.015^{*}$ \\
\hline & 1 & $32(45.7)$ & $17(24.3)$ & \\
\hline & 2 & $23(32.9)$ & $33(47.1)$ & \\
\hline & 3 & $5(7.1)$ & 13 (18.6) & \\
\hline \multicolumn{5}{|l|}{ Late } \\
\hline \multirow[t]{4}{*}{ Xerostomia } & 0 & $38(54.3)$ & $19(27.1)$ & $<0.001^{*}$ \\
\hline & 1 & $29(41.4)$ & $31(44.3)$ & \\
\hline & 2 & $3(4.3)$ & $18(25.7)$ & \\
\hline & 3 & 0 & $2(2.9)$ & \\
\hline \multirow[t]{3}{*}{ Dysphagia } & & $61(87.1)$ & $49(70.0)$ & $0.008^{*}$ \\
\hline & 1 & 9 (12.9) & $15(21.4)$ & \\
\hline & 2 & 0 & $6(8.6)$ & \\
\hline
\end{tabular}

Values are presented as number (\%). INRT, ipsilateral neck radiotherapy; BNRT, bilateral neck radiotherapy. *Statistical significance $(\mathrm{p}<0.05) .{ }^{\mathrm{a}}$ Radiation Therapy Oncology Group acute and late morbidity scoring criteria, ${ }^{\mathrm{b}}$ Chi-square or Fisher exact test.

Table 3. Salvage treatment in patients with a locoregional recurrence $(n=9)$

\begin{tabular}{|c|c|c|c|c|c|c|c|c|c|}
\hline No & $\begin{array}{c}\text { Sex/Age } \\
(y r)\end{array}$ & Stage & CCRT & $\mathbf{R T}^{\mathrm{a})}$ & $\begin{array}{c}\text { Total } \\
\text { dose }(G y)\end{array}$ & Location of recurrence & $\begin{array}{l}\text { Time to } \\
\text { recurrence (mo) }\end{array}$ & $\begin{array}{l}\text { Treatment for } \\
\text { recurrence }\end{array}$ & $\begin{array}{c}\text { Last } \\
\text { status }\end{array}$ \\
\hline 1 & $\mathrm{M} / 52$ & cT2N0 & $\mathrm{N}$ & INRT & 64.8 & Local & 7 & None & NA \\
\hline 2 & $\mathrm{M} / 54$ & cT2N2b & $\mathrm{N}$ & BNRT & 60.0 & Local & 3 & Surgery & CR \\
\hline 3 & $\mathrm{M} / 65$ & cT2N2a & $\mathrm{N}$ & INRT & 66.0 & Local & 27 & Surgery & CR \\
\hline 4 & $\mathrm{M} / 51$ & cT2N2b & Y & INRT & 60.0 & Local & 6 & Surgery & CR \\
\hline 5 & $\mathrm{M} / 51$ & cT2N2b & Y & INRT & 60.0 & Local+regional (contralateral) & 20 & $\mathrm{OP}+\mathrm{reRT}+\mathrm{CT}$ & CR \\
\hline 6 & $\mathrm{M} / 47$ & cT2N2b & $\mathrm{N}$ & INRT & 63.0 & Local+regional (ipsilateral) & 5 & $\mathrm{CT}$ & PD \\
\hline 7 & $\mathrm{M} / 78$ & cT1N2b & $\mathrm{N}$ & BNRT & 63.0 & Regional (ipsilateral)+distant & 12 & $\mathrm{CT}$ & PD \\
\hline 8 & $\mathrm{M} / 53$ & $\mathrm{cT} 2 \mathrm{~N} 2 \mathrm{~b}$ & Y & INRT & 63.0 & Local+regional (bilateral) & 51 & None & NA \\
\hline 9 & $\mathrm{M} / 67$ & cT2N2b & $\mathrm{N}$ & INRT & 54.0 & Regional (contralateral) & 22 & Surgery & CR \\
\hline
\end{tabular}

CCRT, concurrent chemoradiation; RT, radiotherapy; N, no; INRT, ipsilateral neck radiotherapy; NA, not available; BNRT, bilateral neck radiotherapy; $\mathrm{CR}$, complete response; $\mathrm{Y}$, yes; $\mathrm{OP}$, operation; reRT, re-radiotherapy; CT, chemotherapy; PD, progressive disease. ${ }^{\text {a) }}$ All patients with locoregional recurrence were treated by three-dimensional conformal radiotherapy.

\section{Toxicities}

Table 2 compares the acute and late toxicities occurring in patients in the INRT and BNRT groups. A statistically significant difference in the occurrence of grade $\geq 2$ acute toxicities was observed between the INRT and BNRT groups $(45.7 \%$ vs. $74.3 \%, \mathrm{p}=0.001)$. Xerostomia was observed three times more frequently in the BNRT group than in the INRT group (38.6\% vs. $12.9 \%, p=0.001)$, and severe oral mucositis (grade 3) was observed in $7.1 \%$ and $18.6 \%$ of patients in the INRT and BNRT groups, respectively $(\mathrm{p}=0.043)$. Among the late toxicities, 20 patients $(28.6 \%)$ in the BNRT group suffered 
persistent xerostomia, compared to only three $(4.3 \%)$ in the INRT group. No grade 3 toxicity was observed in either group, but six patients $(8.6 \%)$ in the BNRT group experienced grade 2 dysphagia. The overall incidence of grade $\geq 2$ late toxicities were $7.1 \%$ in INRT and $18.6 \%$ in BNRT $(\mathrm{p}<$ 0.001).

\section{Salvage treatment after locoregional recurrence}

Nine patients experienced locoregional recurrence; five of them were salvaged successfully with surgery or combined treatment (Table 3). Two patients died of progressive disease, despite chemotherapy. No information on the treatment after recurrence was available for the remaining two patients because they were loss to follow-up.

\section{Discussion}

Although current consensus recommendations [16] suggested bilateral prophylactic neck irradiation for $\mathrm{N} 2 \mathrm{~b}$ or more advanced disease, the data from a cohort of patients, $>50 \%$ of whom had N2b disease, showed that INRT provides favorable outcomes with less RT-related toxicity in patients with tonsillar carcinoma. In this study, the 5-year LRRFS of patients receiving INRT was $88.1 \%$, and contralateral neck recurrence was $4.3 \%$. These results are similar to those reported in previous studies using INRT for the treatment of cancer of the tonsillar region [7-11]. As one of the largest series evaluating INRT for sparing the uninvolved contralateral neck, O'Sullivan et al. [11] reported that INRT resulted in a 3-year local control rate of 77\% and 3.5\% contralateral regional recurrence in 228 patients with carcinoma of the tonsillar region. In their study, six out of eight patients, whose primary lesion involved the palate or base of the tongue, showed contralateral neck failure, and they suggested that disease confined to the mucosa and with no more than $1 \mathrm{~cm}$ of medial extension could be treated safely by INRT. Since that report, several studies have supported INRT as an effective approach in selected patients without N3 disease. Chronowski et al. [8] reported excellent outcomes of INRT in a limited number of patients $(n=102)$ with TX-2 and NX-2b with low rates of contralateral neck nodal relapse $(2 \%)$ and perfect locoregional control (5-year rate $100 \%)$. Other studies including T3 disease also suggested favorable outcomes, and reported contralateral recurrence rates of $1 \%-6 \%$ and 5-year locoregional control of $90 \%-94 \%$ $[7,10]$.

Our data indicated a borderline significant difference in LRRFS between the INRT and BNRT groups in the PSM pop- ulation (88.1\% vs. 97.1\%, $\mathrm{p}=0.083)$. In the BNRT group, localregional recurrence occurred in two patients (one local and one regional and distant). In the INRT group, local-regional recurrence occurred in seven patients (three local, three localregional, and one isolated contralateral). Of these six patients with local recurrence, two patients showed out-of-field local failure and the remaining four patients had in-field failure. The difference in LRRFS may be due to the difference in radiation modality, in which only five patients $(7 \%)$ in the INRT group were treated with IMRT compared to 31 patients $(44 \%)$ in the BNRT group. Delivering a homogeneous radiation dose to the target volumes including the cervical lymphatic area by 3D-CRT is an inherent challenge. McBride et al. [17] reported that better local control can be achieved using IMRT for the treatment of oropharyngeal cancer. They revealed a lower locoregional failure rate for IMRT compared to conventional RT (1.3\% vs. $10.7 \%, \mathrm{p}=0.017)$. The other advantage of IMRT includes its ability to deliver a higher dose to the target volume with a limited dose to the normal organs. In this study, the median prescribed dose in eight patients with locoregional recurrence (excluding one isolated contralateral regional recurrence among total nine patients) was $63 \mathrm{~Gy}$ (range, 60 to $66 \mathrm{~Gy}$ ), which was similar to the $63 \mathrm{~Gy}$ (range, 50 to $72 \mathrm{~Gy}$ ) in the BNRT group. The median PTV doses of four patients with in-field local failure in the INRT group were 60.0, 63.0, 63.0, and 64.8 Gy, respectively. Therefore, a higher local failure rate in the INRT group might have been related to other factors, such as the inhomogeneous dose distribution, rather than the prescription dose. Considering that the major pattern of failure was local recurrence in the INRT group, where 3D-CRT were used in the majority of patients, the wider adoption of IMRT in recent years may improve local control in patients treated with INRT.

The RT modality (3D-CRT vs. IMRT) could have been used as a variable for PSM to minimize bias. On the other hand, 3D-CRT was covered by the National Health Insurance in Korea during the period of this study, whereas IMRT was not. Therefore, 3D-CRT was used more often in relatively simple INRT, whereas IMRT was used more frequently for BNRT. Hence, there might be a discrepancy in the socio-economic status between the two groups, which could affect the clinical outcome. Considering that all potential unfavorable compounding factors were potentially more prevalent in the INRT group, the 5-year OS rate in this group was not inferior to that in the BNRT group (92.8\% vs. $94.0 \%, \mathrm{p}=0.985)$. This is partly due to the successful salvage treatment; of five patients receiving salvage treatment after locoregional recurrence in the INRT group, four $(80 \%)$ achieved a complete response.

Subgroup analysis of the patients with $\mathrm{N} 2 \mathrm{~b}$ revealed considerable differences in the 5-year LRRFS (84.8\% vs. $94.3 \%$, 
$\mathrm{p}=0.234)$ and contralateral neck recurrence $(7.9 \%$ vs. $0.0 \%$, $\mathrm{p}=0.107$ ) between the INRT and BNRT groups. If the analyses were performed with a larger cohort, it might have reached statistical significance. On the other hand, the risks of contralateral neck recurrence are still small $(7.9 \%)$ with only one patient with an isolated contralateral neck, which was salvaged successfully by a surgical resection. Therefore, INRT for patients with $\mathrm{N} 2 \mathrm{~b}$ can still be offered cautiously for patients who are fit for BNRT.

A HPV infection is associated with favorable outcomes (locoregional control and survival) [13]. Unfortunately, information on the HPV infection status was limited to a small subset of patients in the present study $(21 / 241,9 \%)$; hence, the importance of HPV as a prognostic factor could not be assessed. In addition, the smoking history is an important prognostic indicator. The data contained a similar distribution of non-smokers between the both groups (INRT $51.4 \%$ vs. BNRT $47.1 \%$, $\mathrm{p}=0.536$ ).

Significant differences in the toxicities during or after RT were observed between the INRT and BNRT groups. The patients in the BNRT group were more likely to have persistent xerostomia and dysphagia compared to those in the INRT group, even though more patients in the BNRT group were treated with IMRT (INRT 7.1\% vs. BNRT $44.2 \%$ ). Only three patients (4\%) in the INRT group experienced grade $\geq 2$ xerostomia, whereas 20 patients $(29 \%)$ in the BNRT group experienced this problem. Moreover, approximately $10 \%$ of patients receiving BNRT experienced grade 2 dysphagia, whereas none of the patients undergoing INRT did. The 3\% rate of xerostomia in this population was modestly superior to that of the $8 \%$ reported by Dan et al. [18] who published the outcomes of INRT for node-positive tonsil cancer with a median follow-up of 37.2 months. IMRT is an advantageous option to reduce xerostomia when treating head and neck cancer because it facilitates the delivery of lower radiation doses to the parotid gland. Vergeer et al. [3] compared IMRT with 3D-CRT with regard to xerostomia in head and neck squamous cell carcinoma and reported that the mean dose to the parotid glands was reduced significantly (27 Gy vs. 43 Gy, $\mathrm{p}<0.001)$. Grade 2 xerostomia was observed less frequently in patients treated with IMRT than 3D-CRT (32\% vs.
$56 \%, \mathrm{p}=0.002)$. The xerostomia rate of $32 \%$ after IMRT treatment was consistent with our finding of $29 \%$ in the BNRT group; however, it was considerably worse than the $4 \%$ of the patients observed in the INRT group. Jellema et al. [6], who evaluated RT-induced xerostomia by comparing INRT with BNRT in head and neck cancer, reported that the salivary function could be recovered to the baseline level by sparing the contralateral neck, being compensated for by hyperfunction of the contralateral parotid. Considering the findings from previous studies, exclusion of the uninvolved contralateral neck from the target volume is a more favorable strategy for maintaining the salivary function after treatment, even though the IMRT was adopted widely for the treatment of head and neck cancer.

The limitations of this study are related to its retrospective nature. INRT was compared with BNRT in terms of the locoregional control and survival in a population adjusted by PSM. Although the heterogeneous distribution of the clinical parameters that could influence the prognosis of patients was balanced after PSM, unknown confounding factors, such as physician selection bias regarding which patients received INRT, could remain.

In conclusion, given the data suggesting comparable outcomes with less toxicity, INRT is an attractive strategy in T1-2N0-2a patients with tonsillar carcinoma, compared to BNRT. For patients with $\mathrm{N} 2 \mathrm{~b}$, there were small risks of contralateral neck recurrence when treated with INRT, but its impact on OS was limited with successful salvage treatment.

\section{Conflicts of Interest}

Conflict of interest relevant to this article was not reported.

\section{Acknowledgments}

This work was supported by a National Cancer Center Grant (NCC-1610440) and the Korean Radiation Oncology Group 11-07.

The authors are grateful to Kyung-Ja Lee, MD, Ki-Mun Kang, MD, Suk Won Park, MD, Jin Hee Kim, MD, Dae Sik Yang, MD, Won Taek Kim, MD, Woong-Ki Chung, MD, Sang Gyu Choi, MD, and Min Kyu Kang, MD for patient accrual.

\section{References}

1. Lim YC, Koo BS, Lee JS, Lim JY, Choi EC. Distributions of cervical lymph node metastases in oropharyngeal carcinoma: therapeutic implications for the N0 neck. Laryngoscope. 2006;116:1148-52.

2. Olzowy B, Tsalemchuk Y, Schotten KJ, Reichel O, Harreus U.
Frequency of bilateral cervical metastases in oropharyngeal squamous cell carcinoma: a retrospective analysis of 352 cases after bilateral neck dissection. Head Neck. 2011;33:239-43.

3. Vergeer MR, Doornaert PA, Rietveld DH, Leemans CR, Slotman BJ, Langendijk JA. Intensity-modulated radiotherapy 
reduces radiation-induced morbidity and improves healthrelated quality of life: results of a nonrandomized prospective study using a standardized follow-up program. Int J Radiat Oncol Biol Phys. 2009;74:1-8.

4. Chao KS, Majhail N, Huang CJ, Simpson JR, Perez CA, Haughey $\mathrm{B}$, et al. Intensity-modulated radiation therapy reduces late salivary toxicity without compromising tumor control in patients with oropharyngeal carcinoma: a comparison with conventional techniques. Radiother Oncol. 2001;61: 275-80.

5. Eisbruch A, Kim HM, Terrell JE, Marsh LH, Dawson LA, Ship JA. Xerostomia and its predictors following parotid-sparing irradiation of head-and-neck cancer. Int J Radiat Oncol Biol Phys. 2001;50:695-704.

6. Jellema AP, Slotman BJ, Doornaert P, Leemans CR, Langendijk JA. Unilateral versus bilateral irradiation in squamous cell head and neck cancer in relation to patient-rated xerostomia and sticky saliva. Radiother Oncol. 2007;85:83-9.

7. Al-Mamgani A, van Rooij P, Fransen D, Levendag P. Unilateral neck irradiation for well-lateralized oropharyngeal cancer. Radiother Oncol. 2013;106:69-73.

8. Chronowski GM, Garden AS, Morrison WH, Frank SJ, Schwartz DL, Shah SJ, et al. Unilateral radiotherapy for the treatment of tonsil cancer. Int J Radiat Oncol Biol Phys. 2012;83:204-9.

9. Jackson SM, Hay JH, Flores AD, Weir L, Wong FL, Schwindt $\mathrm{C}$, et al. Cancer of the tonsil: the results of ipsilateral radiation treatment. Radiother Oncol. 1999;51:123-8.

10. Lynch J, Lal P, Schick U, Nutting CM, Newbold K, Harrington $\mathrm{K}$, et al. Multiple cervical lymph node involvement and extracapsular extension predict for contralateral nodal recurrence after ipsilateral radiotherapy for squamous cell carcinoma of the tonsil. Oral Oncol. 2014;50:901-6.

11. O'Sullivan B, Warde P, Grice B, Goh C, Payne D, Liu FF, et al. The benefits and pitfalls of ipsilateral radiotherapy in carcinoma of the tonsillar region. Int J Radiat Oncol Biol Phys. 2001;51:332-43.

12. Sturgis EM, Ang KK. The epidemic of HPV-associated oropharyngeal cancer is here: is it time to change our treatment paradigms? J Natl Compr Canc Netw. 2011;9:665-73.

13. Ang KK, Harris J, Wheeler R, Weber R, Rosenthal DI, NguyenTan PF, et al. Human papillomavirus and survival of patients with oropharyngeal cancer. N Engl J Med. 2010;363:24-35.

14. Fakhry C, Gillison ML. Clinical implications of human papillomavirus in head and neck cancers. J Clin Oncol. 2006;24: 2606-11.

15. Cox JD, Stetz J, Pajak TF. Toxicity criteria of the Radiation Therapy Oncology Group (RTOG) and the European Organization for Research and Treatment of Cancer (EORTC). Int J Radiat Oncol Biol Phys. 1995;31:1341-6.

16. Expert Panel on Radiation Oncology-Head \& Neck Cancer, Yeung AR, Garg MK, Lawson J, McDonald MW, Quon H, et al. ACR Appropriateness Criteria(R) ipsilateral radiation for squamous cell carcinoma of the tonsil. Head Neck. 2012;34: 613-6.

17. McBride SM, Parambi RJ, Jang JW, Goldsmith T, Busse PM, Chan AW. Intensity-modulated versus conventional radiation therapy for oropharyngeal carcinoma: long-term dysphagia and tumor control outcomes. Head Neck. 2014;36:492-8.

18. Dan TD, Raben D, Schneider CJ, Hockstein NG, Witt RL, Dzeda $\mathrm{M}$, et al. Freedom from local and regional failure of contralateral neck with ipsilateral neck radiotherapy for node-positive tonsil cancer: updated results of an institutional clinical management approach. Oral Oncol. 2015;51:616-21. 